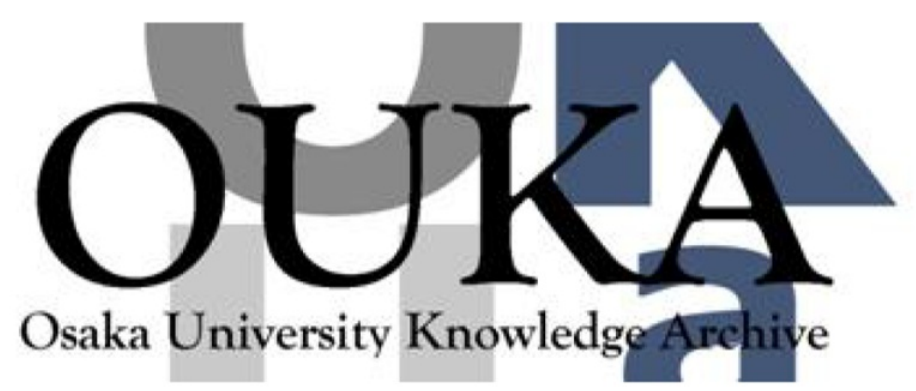

\begin{tabular}{|c|l|}
\hline Title & $\begin{array}{l}\text { Electrically tunable waveguide laser based on } \\
\text { ferroelectric liquid crystal }\end{array}$ \\
\hline Author(s) & $\begin{array}{l}\text { Kasano, Masahiro; Ozaki, Masanori; Yoshino, } \\
\text { Katsumi }\end{array}$ \\
\hline Citation & Applied Physics Letters. 82(23) p. 4026-p. 4028 \\
\hline Issue Date & $2003-06-02$ \\
\hline oaire:version & VoR \\
\hline URL & https://hdl. handle.net/11094/75830 \\
\hline rights & \\
\hline Note & \\
\hline
\end{tabular}

Osaka University Knowledge Archive : OUKA

https://ir. Library. osaka-u. ac. jp/

Osaka University 


\section{Electrically tunable waveguide laser based on ferroelectric liquid crystal}

Cite as: Appl. Phys. Lett. 82, 4026 (2003); https://doi.org/10.1063/1.1580992

Submitted: 10 October 2002 . Accepted: 08 April 2003 . Published Online: 02 June 2003

Masahiro Kasano, Masanori Ozaki, Katsumi Yoshino, Dirk Ganzke, and Wolfgang Haase

ARTICLES YOU MAY BE INTERESTED IN

STIMULATED EMISSION IN A PERIODIC STRUCTURE

Applied Physics Letters 18, 152 (1971); https://doi.org/10.1063/1.1653605

The diverse world of liquid crystals

Physics Today 60, 54 (2007); https://doi.org/10.1063/1.2784685

Flexible mirrorless laser based on a free-standing film of photopolymerized cholesteric liquid crystal

Applied Physics Letters 81, 3741 (2002); https://doi.org/10.1063/1.1522498

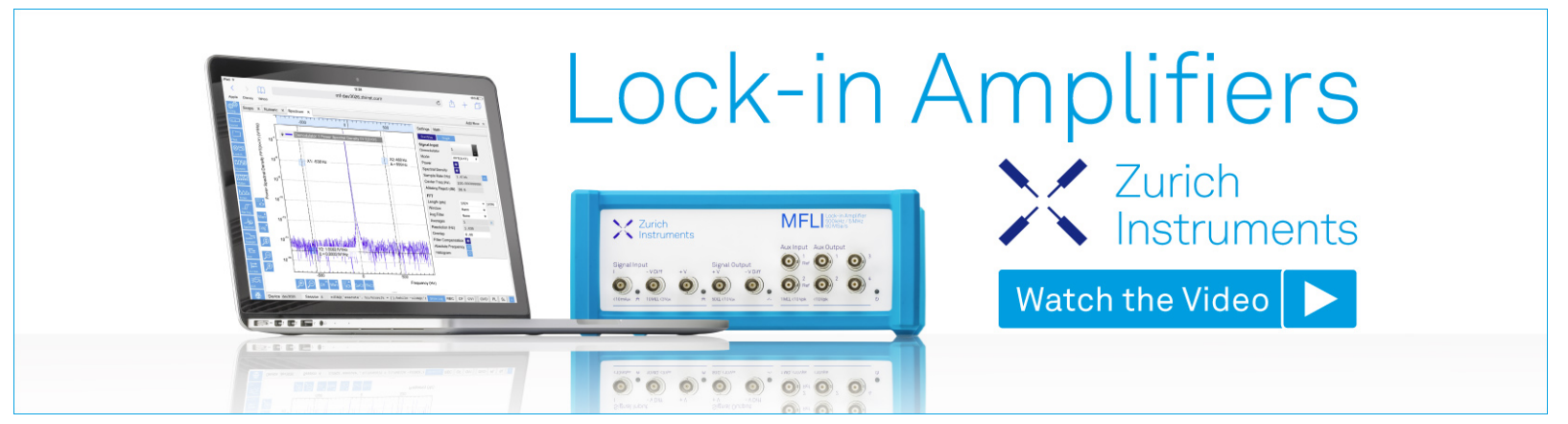




\title{
Electrically tunable waveguide laser based on ferroelectric liquid crystal
}

\author{
Masahiro Kasano, Masanori Ozaki, ${ }^{a)}$ and Katsumi Yoshino \\ Department of Electronic Engineering, Graduate School of Engineering, Osaka University, \\ 2-1 Yamada-Oka, Suita, Osaka 565-0871, Japan \\ Dirk Ganzke and Wolfgang Haase \\ Institute of Physical Chemistry, Darmstadt University of Technology, Petersenstr. 20, Darmstadt 64287, \\ Germany
}

(Received 10 October 2002; accepted 8 April 2003)

\begin{abstract}
Laser action in a waveguide configuration has been demonstrated in a planar alignment cell of dye-doped chiral smectic liquid-crystal mixtures with a short pitch helical structure. In this configuration, doped dye can effectively be excited by a pump beam illuminating perpendicularly the helical axis and the laser light emitted along the helical axis propagates in the waveguide. Lasing wavelength can be tuned by adjusting the periodicity of the helical pitch upon applying the electric field. (C) 2003 American Institute of Physics. [DOI: 10.1063/1.1580992]
\end{abstract}

Photonic crystals having a three-dimensional ordered structure with a periodicity of an optical wavelength have attracted considerable attention from both fundamental and practical points of view, because physical concepts, such as a photonic band gap, have been theoretically predicted and various applications have been proposed. ${ }^{1-9}$ Also, in a onedimensional periodic structure, the laser action has been expected at the photonic band edge where the photon group velocity approaches zero. ${ }^{10}$

Liquid crystals, including chiral molecules such as cholesteric and chiral smectic liquid crystals, have selforganized helical structures. In such chiral liquid crystals with the helical structure, the light propagating along the helical axis is selectively reflected depending on the polarization states (right- or left-hand sided) if the wavelength of the light matches the optical pitch of the helical structure, which is a so-called selective reflection. The wavelength region in which the light cannot propagate is the stop band, which is considered as a one-dimensional pseudoband gap. Lasing at the band edge has been reported in the cholesteric, ${ }^{11-14}$ chiral smectic $^{15,16}$ and polymeric cholesteric liquid crystals. ${ }^{17-19}$

Chiral smectic liquid crystals with tilted structure show a ferroelectricity, which is called ferroelectric liquid crystal (FLC), and have an expected potential for the electro-optic applications because of the fast response to the electric field. ${ }^{20}$ The helical structure of FLC can be easily deformed upon applying electric field and the helical pitch can be changed with a response time of the order of microsecond. Namely, the lasing wavelength can be expected to be controlled quickly upon the electric field.

Lasing in the liquid crystals has been performed in the cell configuration in which a helical axis is perpendicular to the substrates and laser light is emitted out perpendicularly to the cell surface. In this configuration, it is not easy to achieve alignment of high quality in a thick cell, so that it is difficult to extend an active region for the lasing. Moreover, a pump beam is absorbed in the vicinity of the interface between

a)Electronic mail: ozaki@ele.eng.osaka-u.ac.jp liquid crystal and substrate, and doped dye in a bulk is not effectively excited.

In this letter, we design a planar cell configuration of dye-doped FLC for lasing and demonstrate optically pumped lasing in a waveguide. In this waveguide liquid-crystal laser, emission wavelength can be widely controlled by applying electric field.

The FLC compound used in this study is a multicomponent mixture having the chiral smectic $\mathrm{C}\left(\mathrm{SmC}^{*}\right)$ phase in a wide temperature range including a room temperature $\left(\sim 0{ }^{\circ} \mathrm{C}\right.$ to $\left.68^{\circ} \mathrm{C}\right)$. A molecular tilt angle and spontaneous polarization at $30^{\circ} \mathrm{C}$ are $26^{\circ}$ and $55 \mathrm{nC} / \mathrm{cm}^{2}$, respectively. As a laser dye doped in the FLC, a Coumarin 500 (Exciton) was used. The concentration of the dye is $0.2 \mathrm{wt} \%$. The sample was filled into a sandwich cell, which consists of two glass plates. In order to obtain a planar cell in which the helical axis is parallel to the glass substrates as in Fig. 1, surfaces of glass substrates were coated with polyimide (AL1254, Japan Synthetic Rubber) and were rubbed unidirectionally. Thin glass plates were used as spacers, whose thickness was about $150 \mu \mathrm{m}$. In order to align the liquidcrystal molecules perpendicularly to the spacers at the interfaces between the spacer and liquid crystal, the edge of spacer glass was coated with polyimide for a homeotropic alignment (JALS-2021-R2, Japan Synthetic Rubber). In order to apply an electric field normal to the helical axis, glass plates coated with an In-Sn oxide were used as substrates.

As an excitation source, second-harmonic light of a regenerative amplifier system based on a Ti:Sapphire laser (Spectra Physics) was used. The pulse width, wavelength, and pulse repetition frequency of the excitation laser beam were $150 \mathrm{fs}, 400 \mathrm{~nm}$, and $1 \mathrm{kHz}$, respectively. The excitation laser beam irradiated the sample perpendicularly to the cell plate and was rectangularly focused using two lenses, concave lens and cylindncal lens, to area of $0.3 \mathrm{~mm}^{2}$ as shown in Fig. 1. The long axis of the irradiated area on the sample is parallel to the helical axis of the FLC. The emission along the helical axis passed through the spacer glass film and went outside the cell. The spectrum of the light emitted through the glass spacer was measured using a charge-coupled- 


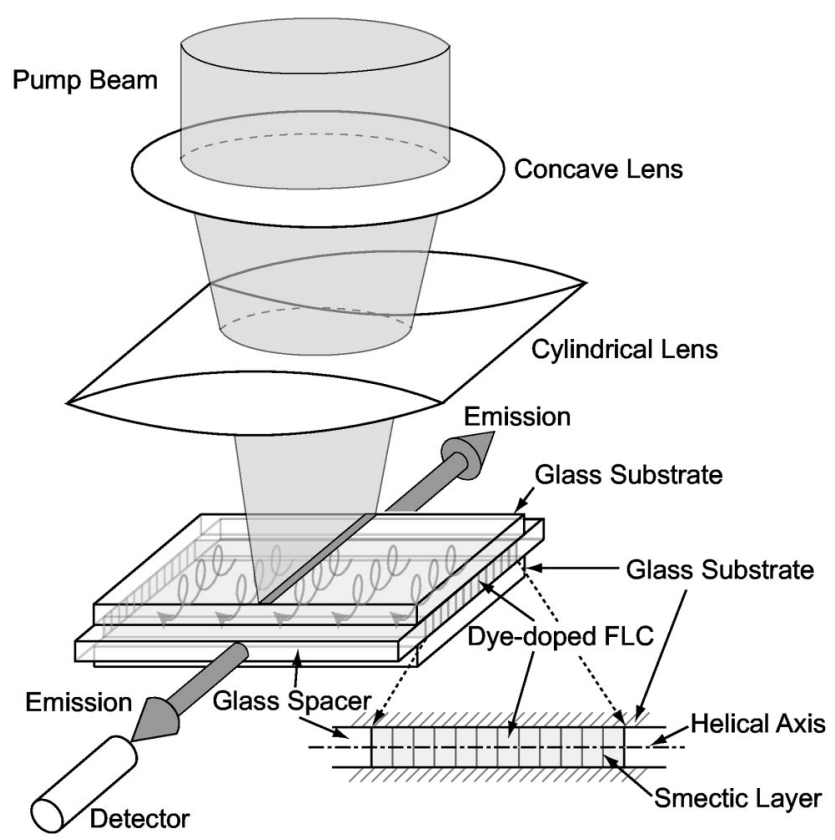

FIG. 1. Schematic diagrams of the cell configurations for the laser action in the liquid crystal.

device (CCD) multichannel photodetector (Hamamatsu Photonics, PMA-11) having spectral resolution of $3 \mathrm{~nm}$. For the measurement with a higher resolution, a spectrograph (Oriel MS257) with a CCD detector having spectral resolution of $0.3 \mathrm{~nm}$ was used. The collecting direction was parallel to both the cell surface and the helical axis as shown in Fig. 1.

Figure 2 shows the emission spectra of the dye-doped FLC as a function of the excitation pulse energy at $22^{\circ} \mathrm{C}$. For low excitation energy $(2.53 \mu \mathrm{J} /$ pulse $)$, the spectrum is dominated by a broad spontaneous emission and the dip is observed in the broad spectrum. The wavelength of the dip shown in Fig. 2 coincides with that of the stop band for the FLC. This dip is wider than that of homeotropically aligned cell. As the excitation energy increases, the emission intensity is enhanced. At high excitation energy ( $36.0 \mu \mathrm{J} / \mathrm{pulse})$, a sharp emission peak appears at the edge of the dip. The full width at half maximum (FWHM) of the emission peak shown in Fig. 2 is about $0.5 \mathrm{~nm}$, which is limited by the spectral resolution of our experimental setup $(0.3 \mathrm{~nm})$. It means that the FWHM of the lasing spectrum at high excitation energy is less than $0.5 \mathrm{~nm}$.

The peak intensity and linewidth of the emission spec-

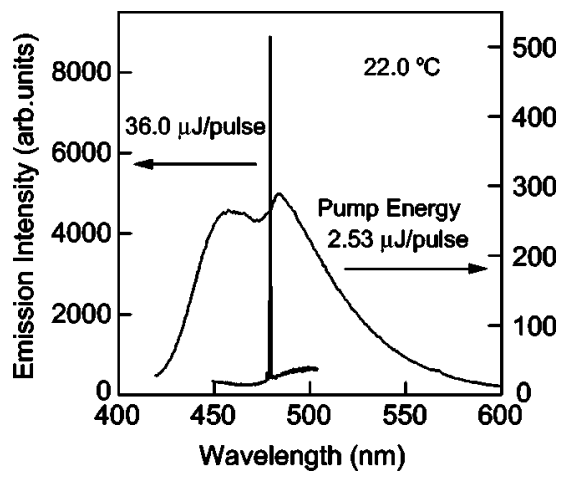

FIG. 2. Emission spectra of a dye-doped FLC as a function of pump pulse energy.

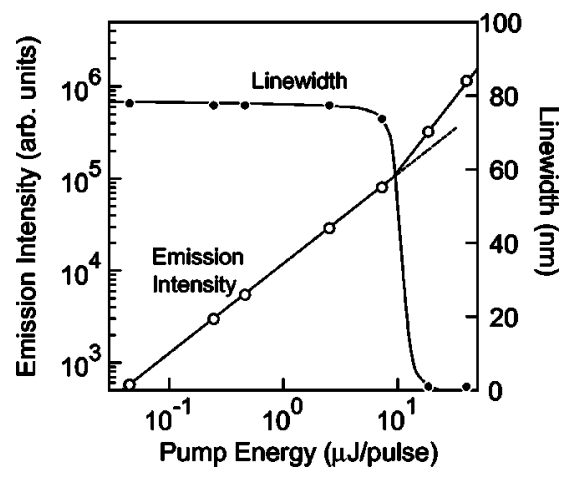

FIG. 3. Pump energy dependence of the peak intensity and linewidth (FWHM) of the emission spectra.

trum are shown in Fig. 3 as a function of the pump pulse energy. There exists a lasing threshold. At lower excitation energy, the emission intensity increases in proportion to the pump energy. Above the threshold at a pump pulse energy of about $10 \mu \mathrm{J} / \mathrm{pulse}$, the emission intensity increases nonlinearly. The linewidth of the emission spectrum also drastically decreases above the threshold. These results confirm that lasing occurs above the threshold of the pump energy at the edge of the photonic stop band in the spontaneous emission. The threshold pump energy for lasing would be lowered by optimizing a doped laser dye, a concentration of the dye, and cell geometries such as thickness and smectic layer arrangement.

In order to confirm the contribution of the helix to the laser action, the temperature dependence of the emission spectra was studied. Figure 4 shows emission spectra below and above the lasing threshold at various temperatures. The dip in spontaneous emission spectrum due to the stop band at low pump energy shifts with temperature, which corresponds to the change in the helical pitch of the host FLC. The helical pitch of FLC used in this study increases with increasing

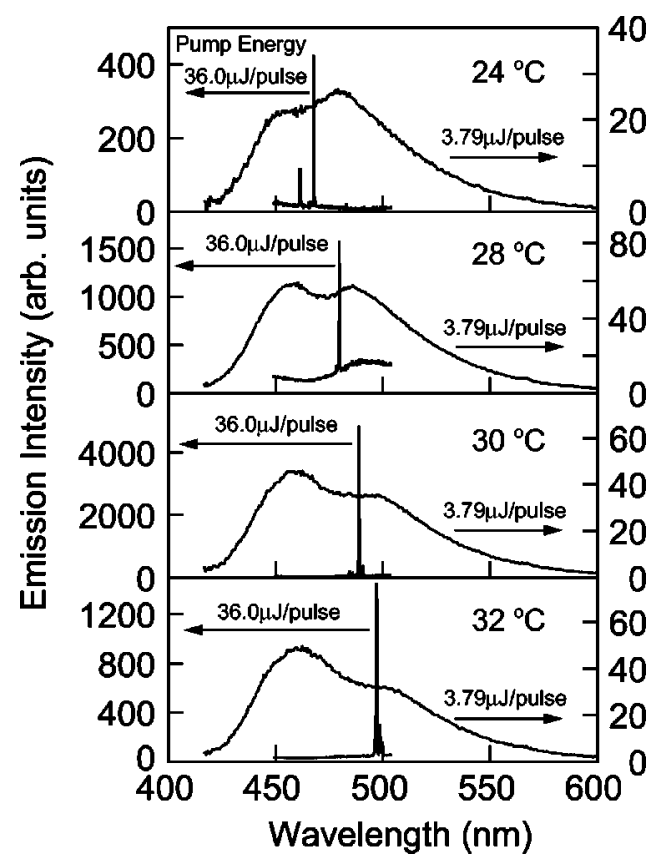

FIG. 4. Temperature dependence of the emission spectra below and above threshold excitation energy for the lasing. 


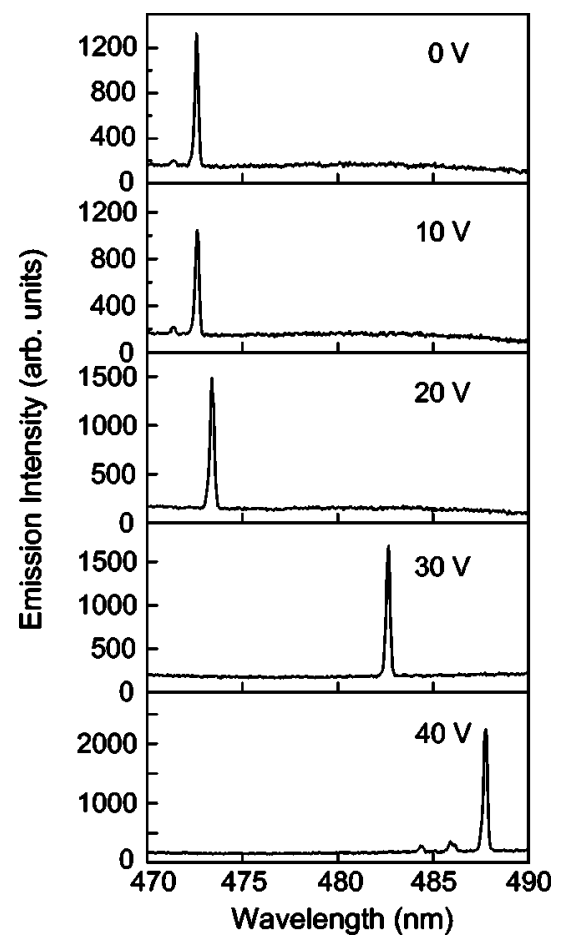

FIG. 5. Laser emission spectra as a function of the voltage applied perpendicularly to the helical axis.

temperature. Therefore, the stop band shifts toward a longer wavelength as the temperature increases.

As is evident from Fig. 4, the lasing wavelength also shifts with temperature according to the shift of the stop band. This clearly indicates that the laser action in the waveguide is based on the periodic structure of the FLC helix.

The helical pitch of the FLC can be controlled also by the electric field, and the FLC should show a fast response of the molecular reorientation to the field because of a strong interaction between spontaneous polarization and the electric field. Figure 5 shows laser emission spectra as a function of the voltage applied perpendicularly to the helical axis. As is evident from Fig. 5, the lasing wavelength shifts toward a longer wavelength with increasing voltage. In spite of a low field $(2.0 \mathrm{kV} / \mathrm{cm})$, a wide tuning of the lasing wavelength was achieved.

FLC has a spontaneous polarization $P_{s}$ which points normal to the molecules and parallel to the smectic layers. In the absence of the electric field, the helical structure is formed. Namely, the long molecular axes of the FLC tilting with respect to the layer normal rotate around the layer normal (helical axis) from one layer to the next. In this structure, $P_{s}$ of the molecules also rotates around the helical axis. When the electric field is applied parallel to the layer, i.e., perpendicular to the helical axis, $P_{s}$ intends to point along the field direction and FLC molecules also intend to orient to the direction normal to the field, resulting in the deformation of the helix and the increase in the helical pitch. The helical pitch monotonously increases as the voltage increases below the critical voltage which completely unwounds the helical structure. The shift of the lasing wavelength shown in Fig. 5 originates from the elongation of helical pitch caused by the field application. Therefore, a high-speed modulation of the laser action is expected using the dye-doped FLC. The applications to the light source of the optical communication and the Q-switched or active mode-locked lasers using the FLC can be expected.

In conclusion, we propose a waveguide laser device based on a dye-doped FLC whose lasing wavelength could be tuned by the electric field. In this device, the axis of the helicoidal periodic structure of FLC is parallel to the substrate and doped dye can be effectively excited by the illumination of the pump beam perpendicularly to the helical axis.

This work is supported by a Grant-in-Aid for Scientific Research from the Japan Ministry of Education, Culture, Sports, Science, and Technology (Grant No. 14350165).

${ }^{1}$ S. John, Phys. Rev. Lett. 58, 2486 (1987).

${ }^{2}$ E. Yablonovitch, Phys. Rev. Lett. 58, 2059 (1987).

${ }^{3}$ J. Martorell and N. M. Lawandy, Phys. Rev. Lett. 65, 1877 (1990).

${ }^{4}$ J. S. Foresi, P. R. Villeneuve, J. Ferrera, E. R. Thoen, G. Steinmeyer, S. Fan, J. D. Joannopoulos, L. C. Kimerling, H. I. Smith, and E. P. Ippen, Nature (London) 13, 143 (1997).

${ }^{5}$ M. Meier, A. Mekis, A. Dodabalapur, A. Timko, R. E. Slusher, J. D. Joannopoulos, and O. Nalamasu, Appl. Phys. Lett. 74, 7 (1999).

${ }^{6}$ M. Imada, S. Noda, A. Chutinan, T. Tokuda, M. Murata, and G. Sasaki, Appl. Phys. Lett. 75, 316 (1999).

${ }^{7}$ K. Yoshino, K. Tada, M. Ozaki, A. A. Zakhidov, and R. H. Baughman, Jpn. J. Appl. Phys., Part 2 36, L714 (1997).

${ }^{8}$ K. Yoshino, S. B. Lee, S. Tatsuhara, Y. Kawagishi, M. Ozaki, and A. A. Zakhidov, Appl. Phys. Lett. 73, 3506 (1998).

${ }^{9}$ Y. A. Vlasov, K. Luterova, I. Pelant, B. Honerlage, and V. N. Astratov, Appl. Phys. Lett. 71, 1616 (1997).

${ }^{10}$ J. P. Dowling, M. Scalora, M. J. Bloemer, and C. M. Bowden, J. Appl. Phys. 75, 1896 (1994).

${ }^{11}$ V. I. Kopp, B. Fan, H. K. Vithana, and A. Z. Genack, Opt. Lett. 23, 1707 (1998).

${ }^{12}$ B. Taheri, A. F. Munoz, P. Palffy-Muhoray, and R. Twieg, Mol. Cryst. Liq. Cryst. 358, 73 (2001)

${ }^{13}$ A. F. Munoz, P. Palffy-Muhoray, and B. Taheri, Opt. Lett. 26, 804 (2001).

${ }^{14}$ W. Cao, A. Munoz, P. Palffy-Muhoray, and B. Taheri, Nature Mater. 1, 111 (2002).

${ }^{15}$ M. Ozaki, M. Kasano, D. Ganzke, W. Haase, and K. Yoshino, Adv. Mater. (Weinheim, Ger.) 14, 306 (2002).

${ }^{16}$ M. Ozaki, M. Kasano, T. Kitasho, D. Ganzke, W. Haase, and K. Yoshino, Adv. Mater. (Weinheim, Ger.) (in press).

${ }^{17}$ H. Finkelmann, S. T. Kim, A. Munoz, P. Palffy-Muhoray, and B. Taheri, Adv. Mater. (Weinheim, Ger.) 13, 1069 (2001).

${ }^{18}$ T. Matsui, R. Ozaki, K. Funamoto, M. Ozaki, and K. Yoshino, Appl. Phys. Lett. 81, 3741 (2002).

${ }^{19}$ J. Schmidtke, W. Stille, H. Finkelmann, and S. T. Kim, Adv. Mater. (Weinheim, Ger.) 14, 746 (2002).

${ }^{20}$ J. W. Goodby, R. Blinc, N. A. Clark, S. T. Lagerwall, M. A. Osipov, S. A. Pikin, T. Sakurai, K. Yoshino, and B. Zeks, Ferroelectric Liquid Crystals (Gordon and Breach, Philadelphia, 1991). 